\title{
RESPUESTA PRODUCTIVA Y ECONÓMICAALUSO DE CUATRO TIPOS DE COMEDEROS PARA FORRAJE EN LA CRIANZA DE CUYES
}

\author{
Productive and Economic Response of Guinea Pigs to the Use of Four \\ TYPES OF FORAGE FEEDERS
}

\author{
Raúl Sánchez V.,4, Ronald Jiménez A. ${ }^{1,5}$, Héctor Huamán U. ${ }^{2}$, José Bustamante L. ${ }^{3}$, \\ Amparo Huamán C. ${ }^{1}$
}

\section{Resumen}

El estudio evaluó el efecto de varias formas de suministro de forraje verde sobre la eficiencia del uso de forraje y la respuesta productiva y económica en la crianza de cuyes (Cavia porcellus). Se trabajó con 250 cuyes machos destetados de 15 días de edad en el valle del Mantaro, Junín, Perú, utilizando un diseño completamente al azar con cinco tratamientos de suministro de forraje, empleando cinco unidades experimentales por tratamiento y cada unidad con 10 cuyes. Se emplearon cuatro tipos de comederos para forraje: de malla sobre el piso en forma cilíndrica (CMP), malla colgante en forma de $\mathrm{U}$ (MCU), parrilla de fierro con patas sobre el piso (PFP) y tolva de malla en forma de V compartida (TMV), además del método tradicional de ofrecer el forraje directamente sobre el piso (DSP). El empleo de los comederos para forraje mejoraron la eficiencia en consumo de forraje, consumo total de alimento y la relación beneficio costo $(\mathrm{p}<0.05)$. El MCU resultó el más promisorio en términos de favorecer la eficiencia en consumo, ganancia de peso, consumo total de alimento, costo de producción unitario y relación beneficio costo $(\mathrm{p}<0.05)$. Se concluye que el uso de comederos beneficiaría productiva y económicamente a las crianzas comerciales de cuyes, siendo el mejor el comedero de malla colgante en forma de U (MCU).

Palabras clave: cuyes, forraje, eficiencia en consumo, comederos

${ }^{1}$ Estación Experimental del Centro de Investigaciones IVITA-El Mantaro, Junín, Perú

${ }^{2}$ Laboratorio de Bioquímica, Nutrición y Alimentación Animal, ${ }^{3}$ Laboratorio de Producción Avícola y Especies Menores, Facultad de Medicina Veterinaria, Universidad Nacional Mayor de San Marcos, Lima, Perú

${ }^{4}$ E-mail: ragu130@hotmail.com

${ }^{5}$ E-mail: ronald_1805@yahoo.es

Recibido: 17 de julio de 2012

Aceptado para publicación: 26 de mayo de 2013 
The study evaluated the effect of various types of forage feeders on the efficiency of forage usage and on the productive and economic response of guinea pig (Cavia porcellus) breeding. A total of 250 weaned male of 15 days of age at the Mantaro valley, Junín, Peru were used in a completely randomized design with five treatments of forage supply and five repetitions per treatment. Each repetition corresponded to a cage with ten guinea pigs. Four forage feeders were evaluated: cylindrical mesh on the floor (CMP), hanging $\mathrm{U}$ shaped mesh (MCU), iron grilled with foot on the floor (PFP) and hopper mesh in $\mathrm{V}$ shaped (TMV), plus the traditional method with the forage directly on the floor (DSP). The use of feeders improved forage consumption efficiency, total food consumption and benefit cost ratio $(\mathrm{p}<0.05)$. MCU was the most promising feeder in terms of forage consumption efficiency, body weight gain, total feed consumption, unit production cost and benefit cost ratio $(\mathrm{p}<0.05)$. It is concluded that the use of feeders improves productive and economic parameters, and the best feeder was the hanging $\mathrm{U}$ shaped mesh (MCU).

Key words: guinea pigs, forage, intake efficiency, feeders

\section{INTRODUCCIÓN}

La crianza de cuyes (Cavia porcellus) viene experimentando desde hace varias décadas una evolución interesante que la califica como una actividad pecuaria económicamente rentable, dada la calidad y demanda de su carne, así como por ser una especie prolífica, de fácil manejo y gran adaptabilidad al medio local y por la baja exigencia del recurso tierra que requiere para su crianza (Chauca, 1999).

El cuy, por ser un animal herbívoro, puede tener un adecuado rendimiento productivo cuando es alimentado básicamente con forrajes verdes de adecuada calidad nutricional (Aliaga, 2006). Así, una cantidad equivalente al $40 \%$ del peso vivo de forraje verde satisface una alta proporción de los requerimientos nutricionales durante la etapa de recría y engorde, aunque puede obtenerse mejores respuestas de ganancia de peso si esta dieta se suplementa con una fuente energética (Jiménez et al., 2000).

La mayoría de los sistemas de crianza de cuyes en el valle del Mantaro están vol- cándose hacia una explotación comercial (Cáceres et al., 2004), la cual conlleva al empleo de una alta proporción de forrajes verdes en la alimentación, dada su disponibilidad y bajo costo a nivel local. Los forrajes más utilizados son la alfalfa (Medicago sativa) y las asociaciones rye grass italiano (Lolium multiflorum) más trébol rojo (Trifolium pratense) y avena (Avena sativa) más vicia (Vicia spp) (Moreno, 1989).

Un aspecto considerarse en el manejo de los forrajes destinados para la alimentación de cuyes en el valle del Mantaro es la forma en que son ofrecidos. Resulta frecuente que, tanto a nivel familiar como comercial, el forraje sea suministrado directamente sobre el piso, sin uso de depósitos. En estos casos, el acceso al forraje puede ser más cómodo para el animal, pero es fácilmente pisoteado y contaminado con heces, siendo rechazado un tercio del forraje ofrecido (Aliaga, 1995). Asimismo, algunos productores restringen exageradamente el alimento forzando al cuy a consumir el total del alimento ofrecido, con el riesgo consiguiente dada la contaminación fecal del forraje (Aliaga, 2006).

El presente estudio tuvo por objetivo la evaluación comparativa de cuatro tipos de 
comederos para suministrar forrajes en términos de ganancia de peso, consumo, índice de conversión alimenticia, costo de producción y relación beneficio-costo.

\section{Materiales y Métodos}

\section{Localización y Animales}

El estudio se realizó entre octubre y diciembre de 2006 en la Estación Experimental El Mantaro del Instituto Veterinario de Investigaciones Tropicales y de Altura (IVITA), ubicado en el km 34, margen izquierda, de la Carretera Central, distrito de El Mantaro, provincia de Jauja, departamento de Junín.

Se emplearon 250 cuyes mejorados en ganancia de peso. Los cuyes eran machos destetados, de pelaje corto y lacio, y con aproximadamente 15 días de edad. Fueron distribuidos aleatoriamente en 25 pozas.

\section{Alimento}

Se empleó un forraje asociado con una proporción similar de trébol rojo y rye grass italiano. El forraje contenía $17.4 \%$ de proteína cruda y 2.72 Mcal de energía digestible, ofrecido a discreción (aproximadamente $60 \%$ del peso vivo en forraje verde). Además, se les suministró agua a discreción en recipientes de arcilla. Como suplemento alimenticio se utilizó la harina de cebada molida a razón de $10 \mathrm{~g} /$ cuy/día, con un aporte nutricional de $2.7 \%$ de proteína y 3.6 Mcal de energía digestible.

\section{Instalaciones y Equipos}

Las pozas de crianza fueron construidas con madera de eucalipto y malla metálica de tejido cuadrangular de $3 / 4$ de pulgada. $\mathrm{El}$ área de cada poza fue de $2.4 \mathrm{~m}^{2}$, compatible con el espacio vital recomendado para la zona de estudio (Cáceres et al., 2004).
El pesaje de los animales y alimentos se hizo con una balanza colgante tipo reloj de $10 \mathrm{~kg}$ de capacidad, con precisión de lectura de $50 \mathrm{~g}$. En el pesaje de muestras para determinación de materia seca se empleó una balanza analítica de $1 \mathrm{mg}$ de sensibilidad.

\section{Tratamientos}

El suministro de forraje se hizo en cinco modalidades (tratamientos):

- DSP, suministro de forraje directamente sobre el piso (Fig. 1)

- CMP, en comederos de malla en forma cilíndrica sobre el piso (Fig. 2)

- PFP, parrilla de fierro con patas (PFP) (Fig. 3)

- MCU, malla colgante en forma de U (Fig. 4)

- TMV, tolva de malla en forma de V compartida (Fig. 5)

Los comederos CMP, MCU y TMV fueron confeccionados empleando malla metálica hexagonal de dos pulgadas. CMP y MCU tuvieron como estructura una varilla de fierro de $1 / 8$ de pulgada y TMV fue a base de madera, mientras que con PFP se empleó fierro de construcción de $1 / 4$ de pulgada.

El comedero TMV funciona como pared y abastece a dos pozas. En este caso, para efecto del estudio, el comedero se dividió entre las dos pozas por igual para efectos de determinar el consumo.

\section{Diseño Experimental}

Se trabajó con un diseño experimental completamente al azar, con cinco repeticiones por tratamiento, donde cada repetición correspondió a una unidad experimental. La poza con 10 cuyes correspondió a una unidad experimental.

\section{Manejo Experimental}

Las pozas fueron limpiadas y desinfectadas antes del experimento. El estudio se inició con un periodo pre-experimental de una 


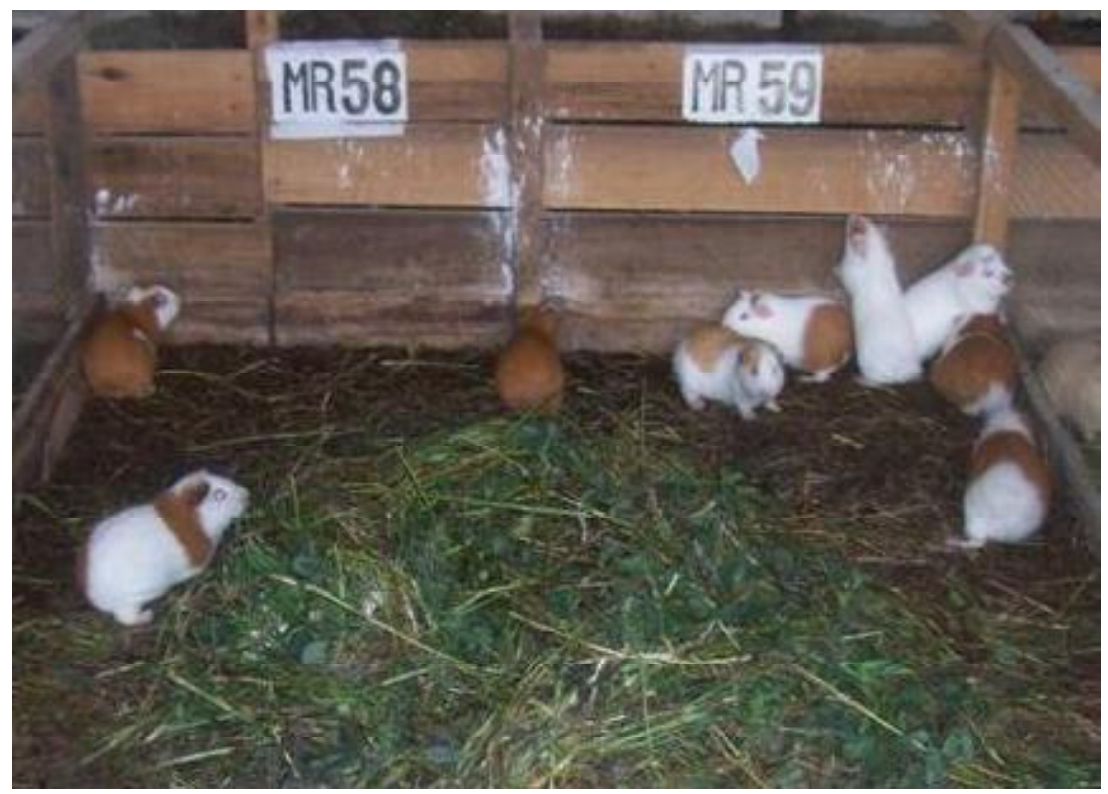

Figura 1. Suministro de forraje sobre el piso (DSP)
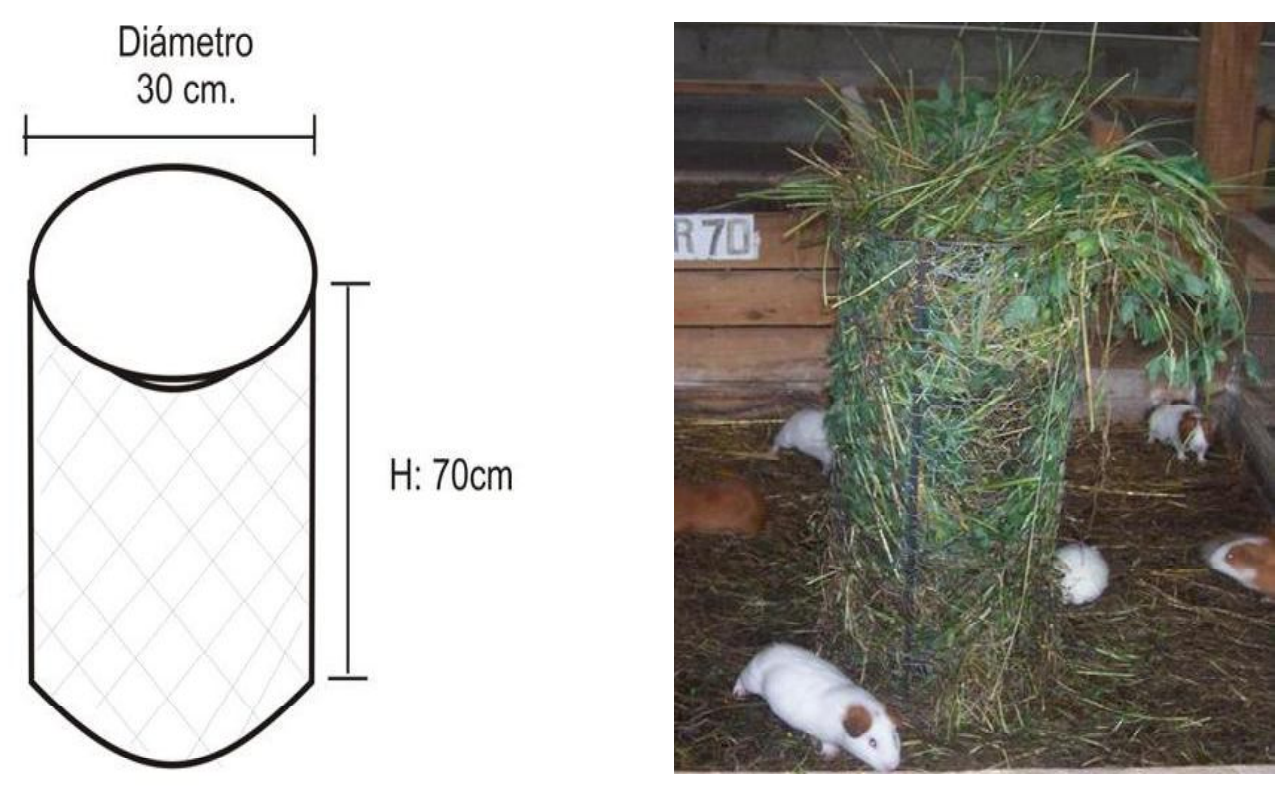

Figura 2. Comedero cilíndrico de malla sobre el piso (CMP) 

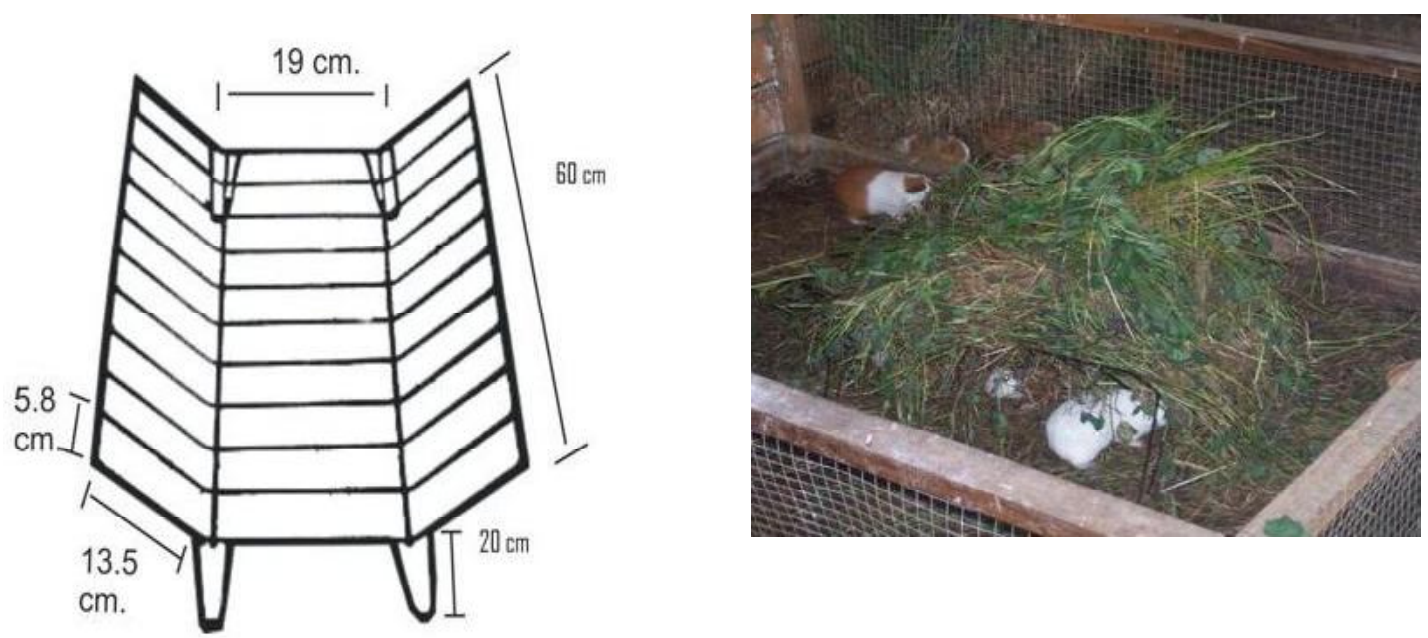

Figura 3. Comedero tipo parrilla de fierro con patas (PFP)
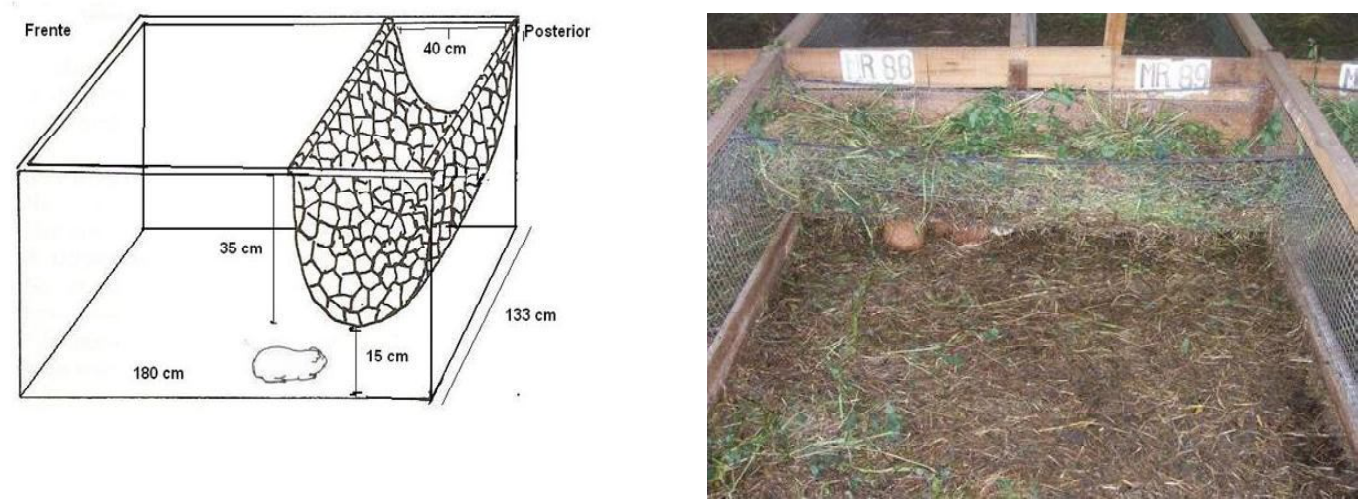

Figura 4. Comedero malla colgante en forma de U (MCU)
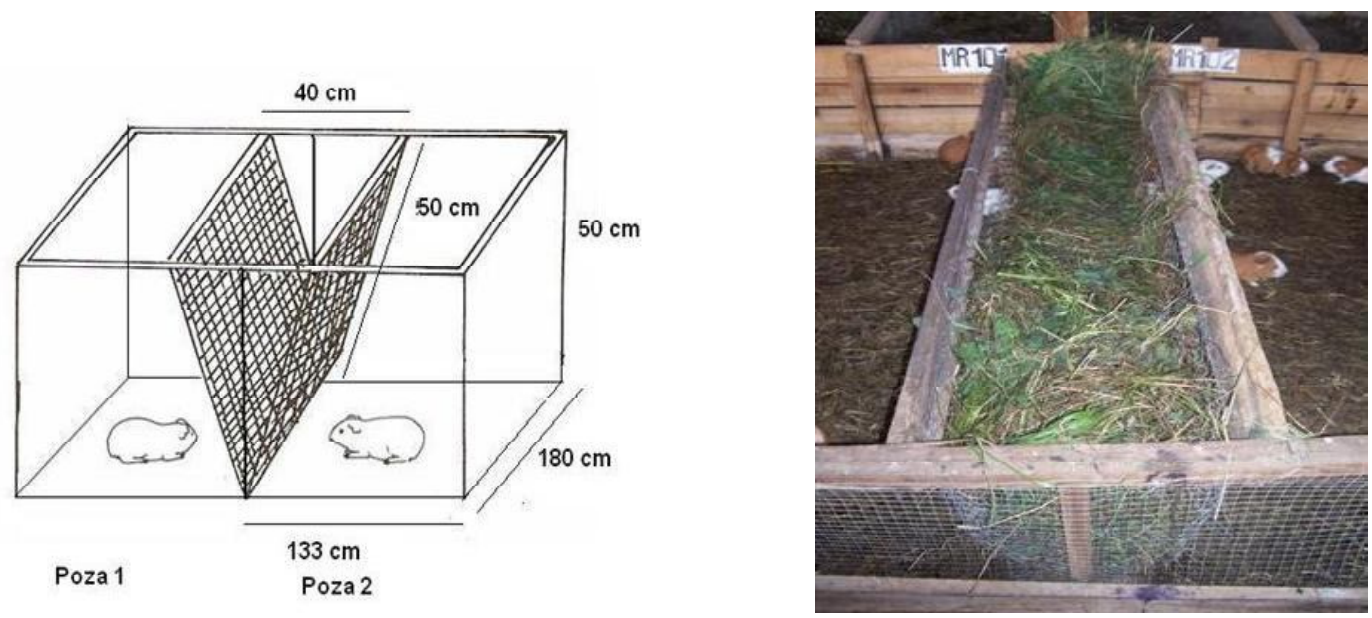

Figura 5. Comedero tipo tolva de malla en forma de V (TMV) 
semana, donde los animales se acostumbraron al tipo de comedero asignado. Asimismo, se evaluó la frecuencia de suministro de forraje más apropiado, estimado por el tiempo en que el comedero reducía su contenido hasta una tercera parte, resultando una vez por día en MCU, una vez cada 1.5 días en TMV, dos veces al día en DSP y PFP y tres veces al día en CMP.

El periodo experimental duró 67 días, tiempo en que los animales alcanzaron el peso comercial de un kilo. Se registró el peso de los animales por unidad experimental al inicio $y$ fin del experimento. El forraje verde se oreaba por 18 horas y se pesaba la cantidad ofrecida. El forraje rechazado o residual retenido en el comedero se recogía y pesaba diariamente. El forraje desperdiciado que se encontraba en el piso era recogido cada tres días y pesado luego de un oreo de $24 \mathrm{~h}$.

Para el recojo del forraje desperdiciado, se colocó en una manta blanca de polietileno de $1 \mathrm{~m}^{2}$ sobre el piso de la poza, por debajo del comedero (o alimento en el caso de DSP). La obtención del alimento desperdiciado requirió de la separación manual de las heces. En DSP no fue posible diferenciar forraje rechazado del desperdiciado y ambos fueron considerados como alimento desperdiciado, dado que estaba en contacto con la cama y estiércol de la poza.

Para la determinación de materia seca se tomaron muestras diarias del forraje ofrecido y rechazado (50 g) y en el caso del forraje desperdiciado se tomaron del acumulado cada tres días. Las muestras frescas fueron pesadas, luego desecadas en una estufa eléctrica a $60{ }^{\circ} \mathrm{C}$ por 48 horas, y nuevamente pesadas.

\section{Variables Evaluadas}

Se determinó la eficiencia en disponibilidad y consumo, así como la ganancia de peso, consumo de alimento, índice de conversión alimenticia (ICA), costo de producción y relación beneficio-costo.
La eficiencia en disponibilidad es la relación porcentual en materia seca del forraje disponible dentro del comedero con respecto al forraje ofrecido. La fórmula es $\mathrm{ED}=((\mathrm{FO}-$ $\mathrm{FD}) / \mathrm{FO}) * 100$, donde: $\mathrm{ED}=$ Eficiencia en disponibilidad, $\mathrm{FO}=$ Forraje ofrecido, $\mathrm{y}$ FD = Forraje desperdiciado.

La eficiencia en consumo es una relación porcentual del consumo neto de materia seca del forraje con respecto al forraje ofrecido. $\mathrm{La}$ fórmula es $\mathrm{EC}=((\mathrm{FO}-\mathrm{FD}-\mathrm{FR}) /$ $\mathrm{FO}) * 100$, donde: $\mathrm{EC}=$ Eficiencia en consumo, $\mathrm{FO}=$ Forraje ofrecido, FD = Forraje desperdiciado, y FR = Forraje rechazado.

La ganancia de peso se determinó por diferencia entre el peso final y el peso inicial de cada unidad experimental, y la ganancia diaria se calculó entre este resultado y los 67 días que duró el ensayo.

El consumo total de alimento se estimó en base seca y fue la diferencia entre el alimento ofrecido como forraje verde y suplemento y el correspondiente a rechazado y desperdiciado. El ICA resultó de dividir el consumo total de alimento entre la ganancia de peso total.

El costo de producción unitario, expresado en soles por cuy, se obtuvo tomando como referencia el módulo de crianza comercial demostrativa de la Unidad en Investigación en Cuyes de la Estación Experimental IVITA El Mantaro, donde se emplea un galpón de $600 \mathrm{~m}^{2}$, 696 reproductoras, 3 ha de pastura, un personal técnico, un obrero, suministro de forraje verde en piso dos veces por día, y suplementación con harina de cebada en el $10 \%$ de la ración, el cual reporta una producción anual de 5568 cuyes para carne. Este sistema tiene un costo total anual de S/. 30,272.00. Sobre este sistema productivo se afectaron las variables del estudio como producción de forraje requerida, mano de obra para el suministro de forraje y costo del comedero, obteniéndose el costo de producción unitario. 
La relación beneficio-costo se obtuvo dividiendo el precio de venta de los cuyes (S/.10.00 por kilo de peso vivo) entre el costo de producción unitario. El beneficio o precio de venta de cada tratamiento se estimó tomando en cuenta un peso de destete a 15 días de edad de $345 \mathrm{~g}$ y la ganancia de peso reportada en el estudio por un periodo de 75 días.

\section{Variables Complementarias}

Adicionalmente, se evaluaron variables que podrían ayudar a explicar las diferencias entre tratamientos. Estas fueron:

- Área de accesibilidad, definida como la superficie por donde el cuy puede acceder a consumir el alimento. Cuanto mayor sea la accesibilidad hay una mayor comodidad para el consumo.

- Ubicación del alimento, está en relación al pasadizo de acceso a la poza (distante, intermedia o cercana). Los cuyes tienden a permanecer más tiempo en el espacio que está distante al pasadizo.

- Frecuencia de suministro. Número de suministros de forraje verde por día.

- Área del orificio en comedero para salida del alimento. En caso de DSP se consideró el área que ocupó el forraje verde en el piso y que fue igual al área de accesibilidad.

- Función madriguera o de ocultamiento, ocurre con animales que tienen depredadores, donde aquellos realizan instintivamente sus actividades, como el consumo de alimento, de una forma más cómoda cuando se encuentran en un lugar donde se sientan ocultos.

\section{Análisis Estadístico}

Las variables eficiencia en consumo de forraje, eficiencia en disponibilidad de forraje, forraje rechazado, forraje desperdiciado, consumo de alimento, ganancia de peso e ICA se evaluaron mediante análisis de varianza, y las diferencias entre promedios se sometieron a la mínima diferencia signifi- cativa de Duncan. El análisis de los datos se efectuó con el paquete estadístico SAS (SAS Institute, 1990).

\section{Resultados y Discusión}

Los tipos de suministros de forrajes mediante comederos tipo MCU y CMP permitieron una mayor eficiencia en disponibilidad y en consumo que DSP ( $\mathrm{p}<0.05$, Cuadro 1 ). La capacidad de cada tratamiento para ofertar forraje verde fue variable entre tratamientos pero no limitante en términos de consumo, pues habiendo la mayor oferta en MCU y la menor oferta en CMP, ambos alcanzaron la mejor eficiencia en consumo de forraje verde $(\mathrm{p}<0.05$, Cuadro 1$)$.

La eficiencia en consumo del forraje verde no parece estar determinada en forma categórica por las variables en estudio, sino por un conjunto de factores adicionales. En el Cuadro 2 se observa que MCU y CMP tienen valores y calificaciones distintas en cuatro de las cinco variables y en la última variable, ambos tienen el menor valor respecto a los otros tratamientos.

El consumo de materia seca total fue mayor en los comederos para forraje respecto a DSP (Cuadro 3), coincidiendo con lo hallado por Peña (1987). Los cuyes de los tratamientos MCU y CMP presentaron mayor consumo de alimento y MCU la mayor ganancia de peso ( $\mathrm{p}<0.05$, Cuadro 3). Asimismo, MCU superó a CMP en consumo total $(\mathrm{p}<0.05)$, pero tuvo una respuesta similar en ganancia de peso; en tanto que los datos de ICA fueron similares entre tratamientos, lo que indica que las variaciones en consumo entre los tratamientos son proporcionales a las variaciones en ganancia de peso. Por otro lado, Peña (1987) evaluó tipos de suministro análogos a MCU, TMV y DSP, y registró valores de consumo y ganancias de peso inferiores a los señalados en el presente trabajo, sin encontrar diferencias estadísticas entre tipos de comedero. 
Cuadro 1. Nivel de oferta y aprovechamiento del forraje suministrado por 67 días en cuatro tipos de comederos o en el piso en crianza de cuyes en pozas

\begin{tabular}{lrrrrr}
\hline \multirow{2}{*}{ Variables } & \multicolumn{5}{c}{ Tratamientos $^{1}$} \\
\cline { 2 - 6 } & MCU & CMP & TMV & \multicolumn{1}{c}{ PFP } & \multicolumn{1}{c}{ DSP } \\
\hline Forraje ofre cido (g MS/poza/día) & $961.9^{\mathrm{a}}$ & $878.8^{\mathrm{e}}$ & $959.0^{\mathrm{b}}$ & $951.6^{\mathrm{c}}$ & $926.2^{\mathrm{d}}$ \\
Forraje rechazado (g MS/poza/día) & $88.3^{\mathrm{b}}$ & $83.3^{\mathrm{c}}$ & $122.2^{\mathrm{a}}$ & $70.5^{\mathrm{d}}$ & $0^{\mathrm{e}}$ \\
Forraje desperdiciado (g MS/poza/día) & $164.4^{\mathrm{d}}$ & $145.7^{\mathrm{e}}$ & $199.1^{\mathrm{c}}$ & $250.2^{\mathrm{b}}$ & $330.9^{\mathrm{a}}$ \\
Forraje verde consumido (g MS/cuy/día) & $70.9^{\mathrm{a}}$ & $65.0^{\mathrm{b}}$ & $63.7^{\mathrm{c}}$ & $63.1^{\mathrm{c}}$ & $59.5^{\mathrm{d}}$ \\
Eficiencia en disponibilidad (\%) & $82.9^{\mathrm{a}}$ & $83.4^{\mathrm{a}}$ & $79.2^{\mathrm{b}}$ & $73.7^{\mathrm{c}}$ & $64.3^{\mathrm{d}}$ \\
Eficiencia en consumo (\%) & $73.7^{\mathrm{a}}$ & $73.9^{\mathrm{a}}$ & $66.5^{\mathrm{b}}$ & $66.3^{\mathrm{b}}$ & $64.3^{\mathrm{c}}$ \\
\hline
\end{tabular}

${ }^{1} \mathrm{MCU}$ : malla colgante en forma de U; CMP: en comederos de malla en forma cilíndrica; TMV: tolva de malla en forma de V compartida; PFP: parrilla de fierro con patas; DSP: suministro de forraje sobre el

$\underset{a, b, c, d, e}{\text { piso }}$ Superíndices diferentes dentro de filas indican diferencias estadísticas $(p<0.05)$

Cuadro 2. Variables de posible influencia en el funcionamiento y consumo de forraje verde de los tipos comederos en cuyes criados en pozas

\begin{tabular}{|c|c|c|c|c|c|}
\hline \multirow{2}{*}{ Variables } & \multicolumn{5}{|c|}{ Tratamientos $^{1}$} \\
\hline & $\mathrm{MCU}$ & CMP & PFP & TMV & DSP \\
\hline $\begin{array}{l}\text { - Área de accesibilidad } \\
\left(\mathrm{m}^{2}\right)\end{array}$ & 0.27 & 0.19 & 0.18 & 0.27 & 0.25 \\
\hline $\begin{array}{l}\text { - Ubicación en relación al } \\
\text { pasadizo de acceso a la } \\
\text { poza }\end{array}$ & Distante & Intermedio & Intermedio & Intermedio & Intermedio \\
\hline $\begin{array}{l}\text { - Frecuencia de } \\
\text { suministro (veces/día) }\end{array}$ & 1 & 3 & 2 & $2 / 3$ & 2 \\
\hline $\begin{array}{l}\text { - Cumple función } \\
\text { madriguera }\end{array}$ & $\mathrm{Si}$ & No & Parcial & No & No \\
\hline $\begin{array}{l}\text { - Tamaño del orificio en } \\
\text { comedero para salida del } \\
\text { forraje }\left(\mathrm{cm}^{2}\right)\end{array}$ & 7.6 & 7.6 & $\begin{array}{l}78.3 \mathrm{y} \\
110.2\end{array}$ & 7.6 & 2500 \\
\hline
\end{tabular}


Cuadro 3. Parámetros de crecimiento y económicos en cuyes empleando cinco tipos de suministro de forraje verde

\begin{tabular}{lrrrrr}
\hline \multirow{2}{*}{ Variables } & \multicolumn{5}{c}{ Tratamientos $^{1}$} \\
\cline { 2 - 6 } & MCU & \multicolumn{1}{c}{ CMP } & TMV & PFP & \multicolumn{1}{c}{ DSP } \\
\hline Peso inicial (g/cuy) & 405.4 & 401.6 & 411.8 & 410.0 & 408.0 \\
Peso final (g/cuy) & $1149.6^{\mathrm{a}}$ & $1116^{\mathrm{ab}}$ & $1080^{\mathrm{bc}}$ & $1099^{\mathrm{b}}$ & $1048.4^{\mathrm{c}}$ \\
Ganancia de peso (g/cuy/día) & $11.1^{\mathrm{a}}$ & $10.7^{\mathrm{ab}}$ & $10^{\mathrm{cd}}$ & $10.3^{\mathrm{bc}}$ & $9.6^{\mathrm{d}}$ \\
Consumo total (g MS/cuy/día) & $79.3^{\mathrm{a}}$ & $73.4^{\mathrm{b}}$ & $72.2^{\mathrm{c}}$ & $71.6^{\mathrm{c}}$ & $68.1^{\mathrm{d}}$ \\
ICA & $7.1^{\mathrm{a}}$ & $6.9^{\mathrm{a}}$ & $7.3^{\mathrm{a}}$ & $7.0^{\mathrm{a}}$ & $7.1^{\mathrm{a}}$ \\
Costo de producción unitario (S/.) & $4.9^{\mathrm{c}}$ & $5.5^{\mathrm{a}}$ & $4.9^{\mathrm{c}}$ & $5.4^{\mathrm{b}}$ & $5.4^{\mathrm{b}}$ \\
Beneficio o precio de venta (S/.) & $11.8^{\mathrm{a}}$ & $11.4^{\mathrm{ab}}$ & $10.9^{\mathrm{cd}}$ & $11.2^{\mathrm{bc}}$ & $10.6^{\mathrm{d}}$ \\
Relación beneficio/costo & $2.4^{\mathrm{a}}$ & $2.1^{\mathrm{c}}$ & $2.2^{\mathrm{b}}$ & $2.1^{\mathrm{c}}$ & $1.9^{\mathrm{d}}$ \\
\hline${ }^{1}$ MCU: malla colgante en forma de U; CMP: en comederos de malla en forma cilíndrica; TMV: tolva de \\
malla en forma de V compartida; PFP: parnilla de fierro con patas; DSP: suministro de forraje sobre \\
el piso \\
a,b,c, Superíndices diferentes dentro de filas indican diferencias estadísticas (p<0.05)
\end{tabular}

Los resultados del costo de producción, beneficio y relación beneficio-costo se presentan en el Cuadro 3. Los tratamientos que obtuvieron el menor costo de producción unitario fueron MCU y TMV, mientras que CMP obtuvo el mayor costo. Los factores que causaron la mayor variabilidad en los costos de producción fueron el forraje, costo del comedero y la mano de obra para el suministro.

En el costo del forraje verde requerido se debe agregar las pérdidas ocasionadas por la cantidad de forraje desperdiciado y rechazado. En este sentido, el tratamiento CMP, seguido de MCU y TMV tuvieron las menores pérdidas y por lo tanto, contribuyeron a un menor costo. Por otro lado, se requiere de casi dos horas hombre para suministrar una rueda de forraje verde a una población de 1000 cuyes (R. Jiménez, Huancayo, comunicación personal), de allí que un mayor número de suministros por día incrementan los costos de producción y esa fue la razón de que CMP, teniendo la menor cantidad de forraje desperdiciado, incrementase su costo al tener el mayor número de suministros (Cuadros 2 y 3 ).

Los resultados de beneficio fueron análogos a lo observados en ganancia de peso, dado que el precio de venta fue en base al kilo de peso vivo del cuy. La relación beneficio-costo favoreció a los tratamientos que emplearon comederos (Cuadro 3), donde MCU obtuvo el mejor valor $(\mathrm{p}<0.05)$.

\section{Conclusiones}

- El uso de comederos mejoran la eficiencia en consumo de forraje, consumo total de alimento y relación beneficio costo.

- El tratamiento MCU resultó el más prometedor por sus efectos beneficiosos en eficiencia en consumo, ganancia de peso, consumo total de alimento, costo de producción y relación beneficio-costo. 


\section{Literatura Citada}

1. Aliaga L. 1995. Importancia de la crianza de cuyes en el ecosistema andino. En: Crianza de cuyes. Lima: INIA. 45 p.

2. Aliaga L. 2006. Crianza de cuyes. Lima: INIA. $97 \mathrm{p}$.

3. Cáceres F, Jiménez A, Ara M, Huamán H, Huamán A. 2004. Evaluación del espacio vital de cuyes criados en pozas. Rev Inv Vet Perú 15: 100-112.

4. Chauca L. 1999. Producción de cuyes (Cavia porcellus) en los países andinos. [Internet], [25 enero 2007]. Disponible en: http://www.fao.org/ag/AGAInfo/ resources/documents/WAR/war/ V6200B/v6200b05.htm
5. Jiménez $R$, Bojórquez C, San Martín F, Carcelén F, Pérez A. 2000. Determinación del momento óptimo económico de beneficio de cuyes alimentados con alfalfa vs. una suplementación con afrechillo. Rev Inv Vet Perú 11(1): 45-51.

6. Moreno R. 1989. Producción de cuyes. $2^{a}$ ed. Lima: Facultad de Zootecnia, Univ Nac Agraria La Molina. 120 p.

7. Peña P. 1987. Uso de comederos para forrajes en casetas diseñadas para la crianza familiar de cuyes. Tesis de Ingeniero Zootecnista. Huancayo: Univ Nacional del Centro del Perú. 83 p.

8. SAS Institute. 1990. User's Guide, v. 6. $4^{\text {th }}$ ed. Cary, NC: SAS Institute Inc. $943 \mathrm{p}$. 